Presented as Invited Talk on September 28, 2004, at the $\mathrm{XXI}^{\text {th }}$ Int. Symposium on Discharges and Electrical Insulation in Vacuum, Yalta, Ukraine.

To be published in Sept. 2005

IEEE Transactions on Plasma Science

Vol. 33 no. 5 (2005)

\title{
The fractal nature of vacuum arc cathode spots ${ }^{\#}$
}

\author{
André Anders \\ Lawrence Berkeley National Laboratory, University of California, \\ 1 Cyclotron Road, Berkeley, California 94720-8223
}

Final version May 27, 2005.

Corresponding Author:

André Anders

Lawrence Berkeley National Laboratory

1 Cyclotron Road

Berkeley, CA 94720-8223

Tel. (510) 486-6745

Fax (510) 486-4374

e-mail aanders@lbl.gov

\footnotetext{
\# This work was supported by the U.S. Department of Energy under Contracts No. DE-AC03-76SF00098 and DE-AC02$05 \mathrm{CH} 11231$. 


\title{
The fractal nature of vacuum arc cathode spots ${ }^{* \#}$
}

\author{
André Anders, Fellow, IEEE
}

Cathode spot phenomena show many features of fractals, for example self-similar patterns in the emitted light and arc erosion traces. Although there have been hints on the fractal nature of cathode spots in the literature, the fractal approach to spot interpretation is underutilized. In this work, a brief review of spot properties is given, touching the differences between spot type 1 (on cathodes surfaces with dielectric layers) and spot type 2 (on metallic, clean surfaces) as well as the known spot fragment or cell structure. The basic properties of self-similarity, power laws, random colored noise, and fractals are introduced. Several points of evidence for the fractal nature of spots are provided. Specifically power laws are identified as signature of fractal properties, such as spectral power of noisy arc parameters (ion current, arc voltage, etc) obtained by fast Fourier transform. It is shown that fractal properties can be observed down to the cutoff by measurement resolution or occurrence of elementary steps in physical processes. Random walk models of cathode spot motion are well established: they go asymptotically to Brownian motion for infinitesimal step width. The power spectrum of the arc voltage noise falls as $1 / f^{2}$, where $f$ is frequency, supporting a fractal spot model associated with Brownian motion.

Keywords: vacuum arcs, cathode spot, fractals, fast Fourier transform, random colored noise

\footnotetext{
${ }^{*}$ Presented as Invited Talk on September 28, 2004, at the XXI ${ }^{\text {th }}$ Int. Symposium on Discharges and Electrical Insulation in Vacuum, Yalta, Ukraine.

A. Anders is with the Lawrence Berkeley National Laboratory, University of California, 1 Cyclotron Road, Berkeley, California 94720-8223 (email: aanders@lbl.gov).

\# This work was supported by the U.S. Department of Energy under Contract No. DE-AC02-05CH11231.
} 
About thirty years ago - I remember it very well - I wondered about the incompatibility of model ideas and experimental facts. What does really happen in the cathode arc spot? At that time, I imagined the great magma lake of the Halemaumau crater in Hawaii, with its red-hot, glowing, molten surface, steaming, boiling, bubbling, gushing, in restless motion, flowing and whirling, rising and falling, covered by clouds of hot vapours, and often throwing gigantic, bright fountains of liquid magma, coupled with ejection of large showers of glowing droplets and pieces and lumps of magma. -- So, similar, as it seemed to me, must be the surface of an arc spot, if we could observe it in action, in quite different time and space scales, of course.

Erhard Hantzsche, in his Dyke Award Address, XXth ISDEIV, Tours, France, 2002

The fractal approach is both effective and "natural." Not only should it not be resisted, but one ought to wonder how one could have gone so long without it.

Benoit Mandelbrot, in: The Fractal Geometry of Nature, 1982

\section{INTRODUCTION}

Cathodic (vacuum) arc spots are known to produce plasma of the cathode material via nonstationary processes [1-3]. Much research has been done over decades to elucidate the nature of the solidto-plasma phase transitions. A coherent picture is only beginning to evolve due to the difficulties associated with the very small scale and very short characteristic times of arc spots, and the complexity of physical processes and environmental conditions.

Cathodic arcs show many features that suggest to model spot phenomena using the well-known theory of fractals $[4,5]$. For example, random walk of cathode spots was discussed in the 1980s [6, 7], 1/f noise of ion current has been found in the late 1980s [8], arc traces by the spot's random walk showed a fractal dimensions of about 2 [9], and self-similarity of cathode spots appearance was recognized [10, 11]. In this work it will be argued that fractal features are not superficial but fundamental to the nature of cathode spots. After reviewing all evidence, one will agree with Mandelbrot's sentiment quoted above. 
The paper is organized as follows. In section II, some features of cathode spot phenomena are described. In section III, a very brief introduction to the world of power laws, self-similarity, and fractals is given. In section IV, a connection is made between cathode spot observations and fractal theory using known and new experimental data. In section $\mathrm{V}$, the role of ignition of emission sites (spot fragments) is emphasized, allowing us to describe spot types and retrograde spot motion in consistent terms. A summary and conclusions can be found at the end.

\section{SOME FEATURES OF CATHODIC ARCS}

Vacuum arc cathode spots have been the subject of research for a long time and have been reviewed several times [12-15]. Since the early observations in the 1930s [16-18], the important role of surface chemistry on ignition conditions was mentioned in the literature many times. Today it is firmly established that spot characteristics depend on surface conditions of the cathode. The distinction between spots of type 1 and 2 was introduced in the 1970s when the role of surface condition on spot formation and operation was fully recognized [19-21]. Unless the cathode is specially treated, e.g. by arcing in ultra-high vacuum, the surface of a cathode has contamination in form of oxides, adsorbates, and dust particles. The presence of contamination reduces the critical field strength for spot ignition [22].

Most of the literature on vacuum arcs focuses on type 2 spots, mainly because the conditions for type 2 are well-defined and therefore better to reproduce. Type 1 spots appear in variety of conditions, depending on the specifics of contamination.

One of the most striking features is that almost all parameters are fluctuating, which becomes quite obvious when one observes motion of spots on the cathode surface. Spot motion is not motion of matter but a process associated with ignition and extinction of active emission sites. Emission refers here mainly to the emission of electrons (the cathode is by definition a source of electrons), but the cathode processes also produce plasma and macroparticles. Fluctuations are found everywhere: in the arc burning voltage, power input, ion production, electron temperature, ion charge states and velocities, macroparticle density and velocity, etc.

Most papers in the literature consider arc noise a nuisance and one tries to come to conclusions by looking at average data. Averaging is done over time, or repeatedly over many discharge events. One can 
find data on average ion charge states [23, 24], average burning voltages [25, 26], average velocities [27], or average velocity distribution functions [28]. Few researchers looked at momentary data and their scattering behavior, like the fluctuation of light [8], voltage [8], current [8], particle fluxes [29, 30], and ion charge states [31].

The difficulties found in measuring fluctuating arc properties are also reflected in the state of theory and simulation. In order to be able to approach the problem, simplifying assumptions need to be made. It turned out to be an art to make reasonable assumptions that do not neglect the essence of cathodic arc physics and still be able to handle systems of coupled integro-differential equations and their boundary conditions. One major school of thought emphasizes the explosive nature, and for modeling one needs to make specific assumptions about the shape and size of microprotrusions. For example, based on the original theory for wire explosions, Mesyats [32] assumed that microexplosions occur as a sequence of explosions of microprotrusions, where the smallest amount of emitted electrons is like a quantum of emission ("ecton"). This approach has mainly been applied to the spot type 2 situation, e.g. [33]. Another major school looks at various zones between the solid cathode and expanded plasma and solves the system magneto-hydrodynamic equations with boundary conditions [34].

In any case, it is difficult to take into account the various stages of development of an emission site, including the actual explosive stage where dense (non-ideal) plasma may be produced. As the experimental technique improved, increasingly finer details of spot structure were revealed. In all spot theories, the issue of current density was central because the current density multiplied by the arc voltage determines the power dissipated, which in turn drives all processes. The issue of current density will be revisited with a fractal interpretation.

\section{A VERY BRIEF INTRODUCTION TO POWER LAWS, RANDOM COLORED NOISE, AND FRACTALS}

Many laws in physics are linear and periodic and show invariance to additive translation. However, not all physical phenomena can be described in this manner, in fact, a great number of phenomena are nonlinear, aperiodic, chaotic. In the 1980s, a branch of mathematics and physics started to flourish: the science of deterministic chaos and self-similar structures, dubbed "fractals" by Benoit 
Mandelbrot [4]. Fractals are invariant to scaling, which makes them "self-similar" to multiplicative changes of scale. A self-similar object appears (approximately) unchanged after increasing or decreasing the scale of measurement and observation. Self-similarity may be discrete or continuous, deterministic or probabilistic.

Power laws are an abundant source of self-similarity [5]. Consider the homogenous power law

$$
f(x)=c x^{\alpha}
$$

where $c$ and $\alpha$ are constants. It is self-similar because rescaling (multiplication with a constant) preserves that $f(x)$ is proportional to $x^{\alpha}$ albeit with a different constant of proportionality. A fruitful approach to fractal modeling is to look for power laws describing the physical phenomena.

Self-similarity can be mathematically exact and infinite or only approximate and asymptotical. The latter applies to the physical world, and scaling cutoffs exist at the small scale and large scale. Mathematical objects are strictly self-similar with infinite scaling; they are often named after their "inventors" and have sometime colorful names, like Cantor sets, Julia sets, Koch flakes, Sierpinski gaskets, Mandelbrot trees, Farey trees, Arnold tongues, Devil's staircases, ... [4, 5, 35].

Fractals can be characterized by dimensional measures, such as the Hausdorff dimension. The fractal dimension is often non-integer and smaller than the embedding topological dimension. To illustrate the concept, Mandelbrot [4] used the now-classical question "How long is the coast of Great Britain?" The essence of this consideration is reproduced here, having in mind that the question of current density of cathode spots should be considered in the same way. Mandelbrot showed that the answer depends on the scale length of measurement, and that different coasts lines have different values for the fractal dimension, depending on their "ruggedness." More detail is revealed by "zooming in," that is, the finer the scale unit $r$, the longer is the apparent coastline length $L$. If the coastline is self-similar, a power law can be found connecting the measured length $L(r)$ with the measuring scale unit $r$,

$$
L(r) \sim r^{\varepsilon},
$$

where $\varepsilon<0$ if $L$ increases as $r$ decreases. In contrast, if the coastline was smooth, the measured length should approach an asymptotic value when a smaller scale is used, $L$ is finite as $r \rightarrow 0$, hence $\varepsilon=0$. The Hausdorff dimension

MS\#TPS0860.R1, -- LBNL-56776 -- 


$$
D=\lim _{r \rightarrow 0} \frac{\log N(r)}{\log (1 / r)}
$$

can be introduced, where $N(r)$ is the minimum number of circular areas of diameter $r$ needed to cover the coastline. In this example, $N(r)=L(r) / r$, leading to

$$
D=1-\varepsilon
$$

Because $\varepsilon<0, D$ will exceed 1, which can be intuitively interpreted as a dimension because a coastline is more than a one-dimensional object (straight line) and less than a two-dimensional object (filled area). Coastlines with only few bays and points have $D$ just little over 1 , while rugged coasts such as the coast of Britain or Norway exceed 1 significantly.

Fourier transform of stochastic, fluctuating data often reveals that the Fourier power spectrum follows a power law

$$
F(P) \sim 1 / f^{\alpha}
$$

where $f$ stands for frequency. According to convention, the exponent $\alpha$ determines the color of random colored noise (RCN, [36]): the noise is white for $\alpha=0$ (i.e., it does not depend on frequency); $\alpha=1$ describes pink noise, which is often found in physical systems of self-organized, barely stable structures [37]; $\alpha=2$ is brown (hinting a relation to Brownian motion); and $\alpha>2$ refers to black noise observed in self-similar systems that tend to have positive feedback, like the stock market.

\section{FRACTAL APPROACH TO CATHODE SPOTS}

Over many years, pieces of evidence for fractal features of cathode phenomena have emerged [611]. At the same time, much research was done in the field of deterministic chaos and fractals $[4,5]$. In this contribution, an attempt is made towards a more comprehensive fractal approach to cathode spot phenomena.

Let us start by considering the pieces of evidence, which include, but are not limited to, (i) random walk of cathode spots, (ii) fractal patterns in multi-spot arc traces, (iii) self-similarity in optical emission patterns, (iv) random colored noise (RCN) in ion current, (v) brown noise in voltage fluctuations, and (vi) a power law distribution of macroparticles. The reader might feel challenged to expand this list.

MS\#TPS0860.R1, -- LBNL-56776 -- 


\section{(i) Random walk of cathode spots}

The spot plasma or the spot "itself" does not move on the surface but the location of ignition of emission sites. Therefore, although the term spot motion is intuitive and reflects a phenomenon that can be visually observed, it is rather abstract because this motion should always be understood as a sequence of ignition and extinction of active emission sites.

The theory and mathematical treatment of random walk has been described many times, for example in the classic paper by Chandrasekhar [38] and text books on stochastic processes [39]. Work by Daalder [7] and Hantzsche and co-workers [6] established that in the absence of an external magnetic field, spot motion may be modeled as random walk. One may assume that each displacement occurs in a small elementary step, $s$, which takes an average elementary time $\tau$. When considering a two-dimensional random walk (i.e., walk on a surface), the probability $P(R)$ for a total spot displacement to be in the interval ( $R, R+d R)$, with $R=|\mathbf{R}|$, measured from the starting point, is given by [40]

$$
P(R) d R=\frac{R}{2 D t} \exp \left(-\frac{R^{2}}{4 D t}\right) d R \text { for } t>>
$$

The diffusion constant, $D$, contains the parameters of the elementary step:

$$
D=\frac{1}{4} \frac{s^{2}}{\tau}
$$

The diffusion constant is material dependent, for example Jüttner found experimentally $D=(2.3 \pm 0.6) \times 10^{-3} \mathrm{~m}^{2} / \mathrm{s}$ for copper [41] and $D \approx 10^{-3} \mathrm{~m}^{2} / \mathrm{s}$ for molybdenum [42]. The mean value for displacement is

$$
\langle R\rangle=\int_{0}^{\infty} R P(R) d R=(\pi D t)^{1 / 2}=\frac{s}{2} \sqrt{\pi \frac{t}{\tau}},
$$

and the observable, apparent spot velocity is

$$
v_{\text {spot }}=\frac{d\langle R\rangle}{d t}=\frac{1}{2} \sqrt{\frac{\pi D}{t}}=\frac{s}{4} \sqrt{\frac{\pi}{t \tau}} .
$$


From (9) one can see that the apparent spot velocity decreases as the observation time increases, which is a consequence of the random nature of this motion.

At this point it should be emphasized that within the random walk model, spot motion is apparent and a velocity is only defined in the sense of the time derivative of the average over many elementary steps, considering changes of displacement for long observation times: $t>\tau$. Strictly speaking, elementary steps are associated with ignition events, and therefore this "motion" itself does not have a derivative, hence a velocity in the usual sense is not defined.

Random walk is a discrete fractal exhibiting stochastic self-similarity on large scales, but selfsimilarity is cut off as scales approach the elementary step width s. The well-known Brownian motion is the scaling limit of random walk. That means that if random walk occurs with very small steps, $s \rightarrow 0$, random walk becomes an approximation to Brownian motion. Brownian motion in two dimensions is a true fractal which means that it shows self-similarity on all scales. Brownian motion has the fractal (Hausdorff) dimension 2. Often, to make modeling and computation more efficient, Brownian motion is approximated by a random walk.

In the literature, random walk of cathode spots has been analyzed with the assumption of a constant elementary step s. Hantzsche and co-workers [6] suggested using the mean crater radius as the elementary step length for type 2 spots based on analysis of arc traces on clean metal surfaces. The assumption is justified by the appearance of crater chains, though its general validity was disputed by Daalder [43] because, so he argued, one can also observe separated craters, which are usually associated with type 1 spots. While in fact surface conditions are decisive for specific events, the argument can be seen in different light when using the fractal approach to spot phenomena. The issue can be reduced to a probabilistic distribution of ignition of emission centers or a fractal understanding in the sense of Brownian motion, where the grid of steps approaches zero.

This discussion of the role of surface conditions leads to an extension of random walk. In a random walk, the next position depends only on the current position of the "walker" (the emission site). By definition, random walk has no memory of all other previous positions, i.e., the walk history. Such process is called a first-order Markov process [5], and the chain of events is called a Markov chain [39]. Provided that cathodic arc processes occur on clean metal surfaces, such as well-arced cathodes, one can 
assume that all sites next to the active site have approximately the same probability to serve as the next emission site, and the walk may be modeled as a Markov process.

More realistic, however, is that the arc changed the surface conditions and local temperature in such a way that the ignition probability distribution is changed. Experiments like the breakdown experiments by Kraft and Stuchenkov [44] showed that arcing on contaminated surfaces is preferred or relatively "easy," leading to type 1 spots, while arcing on metallic, clean surfaces is relatively "difficult" (type 2 spots). Loosely speaking, the arc "prefers" to burn with type 1 spots. Of course, the arc has no opinions and strictly speaking one should state that the ignition probability for sites with non-metallic layers or other dielectrics (like a dust particle) is higher than the ignition probability of a location with clean metal surface. Therefore, the cleaning action of arc erosion will markedly reduce the ignition probability of locations where the arc has already burned.

If we consider an oxidized or otherwise contaminated cathode, the random walk will turn into in special class of self-interacting walk, the self-avoiding random walk [39, 45]. As a result of arc cleaning, the next emission is much more likely to be ignited at locations that still have the surface-field-enhancing oxide layer rather than on the arc-cleaned surface. As long as there is surface area with oxide layer (or similar contamination) available, emission sites will avoid the cleaned surfaces. This feature has been utilized in arc cleaning of steel surfaces where oxide surfaces were intentionally created to optimize cleaning effects [46].

If arcing occurs in reactive gas environment or poor vacuum, the surface will quickly "age" by becoming "poisoned," i.e., the metal atoms on the cathode surface will reacted with oxygen or water from the residual gas. In this way, the surface will "forget" its memory on the local emission event, and the walk may become Markovian rather than self-avoiding, but this time with type 1 spots.

If arcing of the cathode surface occurs in ultrahigh vacuum (UHV), or in a noble gas free of any reactive components, prolong arcing may eventually completely remove the oxide layer. As a consequence, all surface locations become equivalent, and in this case the self-avoiding walk will switch to a random (Markovian) walk with type 2 spots. 
Therefore, in both extreme cases, arcing with reactive gas and arcing in UHV, there is a tendency to go from random walk through a phase of self-avoiding walk, which eventually may be followed by another phase of random walk.

This picture may however be even more complicated. Not all self-interacting random walks are self-avoiding walks [39]. For example, investigating clean, well-arced cathodes, it has been observed that after a short cool down time, a previously active and still hot emission site may preferably re-ignite [47]. In this case, the walk is not self-avoiding but rather shows a preference to return to a familiar location. Apparently, research is not completed. The fractal approach may provide a helpful framework for the multitude of individual situations.

(ii) Fractal patterns in multi-spot arc traces,

Closely related to random walk discussed above are patterns left on cathodes by multi-spot discharges. An example of such pattern is shown in Fig. 1, which is strikingly similar to patterns wellinvestigated in fractal research, such as Lichtenberg figures and macroclusters obtained by diffusionlimited aggregation [48]. The multi-spot arc patterns occur not only on massive cathodes but have also been observed on film cathodes. For example, analyzing arc traces on an aluminum film made a by a $50 \mathrm{~A}$ arc, Anders and Jüttner [9] found a fractal dimension of 2 within 5\%, which is the expected fractal dimension for two-dimensional Brownian motion.

(iii) Self-similarity in optical emission pattern

Naturally, if fractal structures are found in arc traces (craters) left on cathode surfaces, one can also expect to see fractal patterns in the light emitted by cathode spots. This turns out true for both spatial light distribution $[10,11]$ and temporal (noise) behavior [8].

\section{(iv) Random colored noise in ion current}

Each emission site is not only a source of electrons but ionized cathode material. Ions have been collected by negatively biased probes placed in some macroscopic distance (for example, see companion paper [49]). Early measurements by Smeets and Schulpen [8] indicated that the Fourier amplitude is 
$\sim 1 / f^{\beta}$ with $\beta \approx 1$, for frequencies up to $15 \mathrm{MHz}$, and $\beta=0$ at higher frequencies. Unfortunately, their display of data was linear and it is hard to judge how well the noise was indeed $\sim 1 / f$. The slope of a power law, if present, is much easier to find when data are displayed in log-log presentation. Furthermore, they did not explicitly distinguished between Fourier amplitude and Fourier power spectrum but we may assume that they referred to the amplitude spectrum. The corresponding power spectrum would be $\sim 1 / f^{\alpha}$ with $\alpha \approx 2$, i.e., brown noise.

Using our own data [49], and analyzing the noise in ion current using Fast Fourier Transform (FFT), we found a linear slope indicating random color noise within the physically meaningful frequency interval. Most data curves show three characteristic segments. There was white noise at high frequencies. At intermediate frequencies, a RCN slope of $\alpha>2$ was found. Many curves had a kink at lower frequencies, giving $0<\alpha<2$. Figure 2 shows such curve for the noise of $\mathrm{Cu}$ ions for a pulsed vacuum arc.

White noise at high frequencies is possibly due to ion velocity mixing [49]. That is, all information from emission sites is mixed because faster ions overtook slower ions on their way from the cathode to the ion collector. "Scrambled" information would result in white noise. In the case of carbon, Fig. 3, the segment of white noise is particularly pronounced. Carbon ions are lighter and faster than other metal ions, and the effect of velocity mixing could be more pronounced. However, such conclusion cannot yet be drawn because the white noise can also be caused by measurement limitations, in particular when the amplitude of the noisy signal approaches the noise of the measuring system itself. Further experiments and modeling are needed.

At its low-frequency end, the curve is quite uncertain due to insufficient data. Mathematically, because we deal with a finite discrete sample of data, the limits of Discrete Fourier Transforms (DFT) apply: The inverse of the sampling period represents a lower limit to the resolution of the transformed data, and the upper limit is half of the sampling interval (Nyquist sampling theorem). Only few data points contribute to the lower frequency part of the FFT curve, and therefore it should be disregarded.

More research is needed to really learn what information can be extracted from the RCN segment of such FFT curves. So far, one can see that the relevant segment appears close to brown noise, another indication for a fractal system where Brownian motion plays a role.

MS\#TPS0860.R1, - - LBNL-56776 -- 
(v) Brown noise in voltage fluctuations

The arc voltage is a special parameter because it reflects cathode processes in a less distorted way than ion current or most other parameters. This is because phase mixing, as it is prevalent with ion currents [49], or "smearing out" of emitted light due to finite, non-zero lifetime of excited states [50], does not play a role. Using voltage noise, the signal generated by the cathode processes can be recorded with maximum fidelity, only limited by the bandwidth of the recording circuit. Using a coaxial discharge arrangement, $250 \mathrm{MHz}$ analog bandwidth and $1 \mathrm{Gs} / \mathrm{s}$ sampling rate, the voltage of a pulsed magnesium arc has been analyzed (Fig. 4). More data and further details are provided in a separate publication [51] and further research is ongoing. One can recognize the $1 / f^{2}$ (brown) noise, which points again to fractal properties and Brownian motion.

\section{(vi) Power law distribution of macroparticles}

In the explosive formation of plasma, craters are formed, which have become a signature of cathode arcs. Closely related to explosive formation of plasma is the formation of (usually unwanted) droplets, commonly called "macroparticles" to emphasize their massive nature compared to ions and electrons. It is well known that smaller macroparticles are much more frequent than large ones. Several investigations quantified this by plotting size distribution functions $[23,52,53]$. Fig. 5 shows data by Siemroth and coworkers [54], which interestingly not only confirms the power law of the size distribution but also indicates that a fractal dimension may be defined, characterizing the dependence of the size distribution from experimental conditions.

In any case, the power law distributions, extending over several orders of magnitude, confirm the self-similar (fractal) property: an observer looking at electron microscope photographs of macroparticles would be unable to decide which magnification was used.

V. DISCUSSION: IGNITION CONDITION, SPOT FRAGMENTS, AND THEIR RELATION TO A FRACTAL SPOT MODEL 
In this section, a cathode spot is described in general terms as a self-organized, dissipative structure containing highly dynamic "fragments" or "cells." The idea of spot cells has been introduced decades ago by Kesaev [12]. Direct evidence for their existence came with high-resolution diagnostics using pulsed lasers $[55,56]$ and image converter cameras [1]. Fragments and their ignition represent possible short-scale, short-time cutoffs for a fractal description of cathode spots, which will be further discussed below.

A spot cell or fragment is, as the name suggests, an emitting structure, or emission site, which is part of what is typically seen as one more-or-less blurred cathode spot. It requires sophisticated diagnostic techniques of high spatial and temporal resolution ( $\mu \mathrm{m}$ and ns, respectively) to investigate fragments [1]. One may assume that each fragment leaves a crater behind, although it seems clear that it takes the action of many fragments to produce large craters. The terms cell and spot fragment are used synonymously, and one may also use emission site to emphasize the function of the structure.

The ignition of emission sites is of greatest interest for understanding of the phenomenology and operation of cathodic arcs. Without introducing much limitation, one may generally state that an arc spot emission site is ignited when a location on the cathode surface surpasses a critical electrical field strength, leading to thermal runaway [57]. The critical surface field is a function of surface conditions and cathode material, and the actual field depends on the arc voltage and local plasma (sheath) conditions.

With this concept, we have an opportunity to develop a unified description of type 1 and type 2 spots by considering the critical field strength involving a broad range of possible surface geometries and material compositions. While critical field and field enhancement are well-described for protrusions of clean metal [58], field enhancement due to charge-up is less accessible to modeling. Experimental evidence of easier ignition of spot fragments on surfaces with dielectric layers points to an enhanced ignition probability due to dielectrics. Many locations on the cathode surface are potentially the location of the next active emission site. The distribution of local critical field strengths could be modeled by a distribution of ignition probabilities.

An active (plasma-emitting) spot fragment will increase its area in time ( $\sim 10-100 \mathrm{~ns}$ timescale) due to heat conduction, and thus the power density, $P \approx j \Delta V$, will decrease because the current density $j$ decreases. Depending on the surface conditions, the following scenarios are possible:

MS\#TPS0860.R1, - - LBNL-56776 -- 
(i) At least one location near the original spot fulfills the condition for exceeding the critical field, and a new emission site is formed that is electrically in parallel to the original site. Taking the hot cathode zone and liquid phase into account, the new site will have less total resistance and therefore provides favorable conditions compared to the parent spot fragment. It will take over the current from the original spot fragment and accelerate the cool-down of the parent.

(ii) If ignition occurs easily, such as in the presence of many sites having an oxide layer [59], many fragments may be ignited that operate electrically in parallel. They compete for available current and associated power dissipation, and their plasma will promote the ignition of yet more emission sites. This will shorten their active stage, leading to small craters, low cathode erosion, low plasma and light emission, etc. Thus favorable ignition conditions provide a straight-forward, still qualitative explanation of type 1 spots.

(iii) No location initially exceeds the critical field conditions. The original spot will still reduce it power density after its original explosive stage due to heat conduction. The strongly nonlinear nature of electron emission, evaporation, and other related processes will cause the spot to ceases to provide sufficient electrons and plasma. The impedance of the discharge increases and the burning voltage will increase accordingly. Its rise may go all the way to the open-circuit voltage of the supply circuit. In the case of low currents, the current may be affected (known as current chopping), and voltages much higher than the open-circuit-voltage of the power supply can be generated due to the inductive response of the arc circuit $[60,61]$. The rising voltage will lead to an increase of the electric field strength on the cathode surface, which, in turn, may lead to the fulfillment of the spot ignition condition [62]. If the ignition condition is still not fulfilled the arc will spontaneously extinguish.

From this discussion, which is further detailed in [15], we can see that the spot undergoes several stages, which may be summarized as (1) the pre-explosion stage, (2) the ignition stage (microexplosion, formation of non-ideal plasma, initial plasma expansion), (3) the hot, emissive stage with decreasing power density, and (4) the cool-down stage, when electron emission becomes negligible but evaporation may be still a factor contributing to overall cathode erosion. Impedance and plasma parameters change rapidly in time, which is evident from many measurements of burning voltage, plasma density, light emission, ion charge state distribution, etc.

MS\#TPS0860.R1, -- LBNL-56776 -- 
Considering the ignition probability and actual field conditions for vacuum arcs in a magnetic field, retrograde motion can be consistently described using the same terms. In the absence of a transverse magnetic field, the probability for ignition is symmetrically distributed around an existing emission site. When a transverse magnetic field is present, the axial symmetry is broken (see e.g. [63]), and one should expect a preferred direction. Jüttner and Kleberg [64] found that the spot plasma shows instabilities leading to ejection of plasma jets around the retrograde direction. The actual surface field is enhanced in the retrograde direction due to the appearance of plasma jets because these jests lead to a locally thinner sheath and an additional $\mathbf{E}=\mathbf{v} \times \mathbf{B}$ field.

Without further reviewing the literature at this point, the relation of ignition of emission sites and fractal spot model should be clarified. A fractal model cannot be used to analyze the elementary physical mechanisms of spot fragments. Rather, the fractal model can help to interpret the variety of experimental data obtained with various methods and resolution. It is clear that the resolution limits are the cutoffs of any fractal property displayed and that true elementary steps, should they exist, are the physical cutoff of a fractal.

For example, one sees leveling-off of the macroparticle distribution on the small-scale side of the distribution in Fig. 5. The measurements over several orders of magnitude in macroparticle diameters were done in several steps of different magnification using an electron microscope and image processing system. Each of the steps resulted in a curve like displayed in Fig. 5 but over only about one order of magnitude, and each of the curves indicated leveling-off on its small-scale side. The limited resolution at a given magnification caused the apparent deviation from the power law, which is the basis for self-similarity. The entire curve was composed by combining all measuring steps, yet its small diameter end still appears to be leveled-off caused by the method rather than true physics. Only if leveling-off was also observed at the same place using even higher resolution, the true cutoff for the physical fractal would have been found.

Locking now at ignition events and their high-resolution recording made to-date, we probably have not yet reached the physical cutoff limits for the fractal. While high-resolution imaging revealed many details, more details may appear if methods with even greater resolution were available. That is, the current limit of resolution represents a display cutoff for fractal properties. Since the existence of fragments or cells is today confirmed, all measurements with less resolution involve a multitude of 
stochastic events, leading to fractal characteristics. One should expect that the corresponding power laws are directly associated with dissipative processes and Brownian motion. In analogy to spatial resolution of macroparticle measurements, true elementary ignition processes are only observed when improved temporal and spatial resolution do not reveal even smaller structures.

From this point of view, we may re-interpret the long-standing discussion on the current density of arc cathode spots. Fig. 6 shows that the current density appeared to be increasing over the years of investigation. Such figure was originally shown in the 1980s with a wink, in the assumption that one eventually would settle on a value as measuring techniques improved. In the framework of a fractal model one may say that a unique value of current density does not exist, similar to the conclusion that a unique value of a coastal length does not exist. The current density of cathode spots should be understood as a fractal. The higher the resolution the greater the value - but the issue is not just a question of spatial resolution. The fractal spot is a dynamic phenomenon, and the fractal concept applies in space and time.

\section{SUMMARY AND CONCLUSIONS}

Vacuum arc cathode spots consist of fragments or cells, which may also be described as emission sites. Until now, no conclusive evidence for a truly "elementary" spot structure has been provided and therefore even smaller physical substructures may exist. Current knowledge is based on the understanding that the physical processes are non-stationary and therefore one may consider various stages of spot fragment development. The onset of electron emission and plasma generation can be described as ignition of an emission site. Ignition occurs when the actual local electric field strength exceeds a critical field strength whose local value depends on cathode material, nano- and micro-scale surface geometry, as well as on dielectric layers, particulates, and other special field-enhancing conditions. The distribution of critical field strength may be modeled by a distribution of ignition probabilities. Ignition occurs much more readily when dielectric layers and "contaminations" are present, and therefore a larger number of spots may ignite, which operate electrically in parallel, competing for the available power. Such situation would describe fragments forming a type 1 spot. In contrast, in the absence of field-enhancing dielectrics, ignition is less probable and power is concentrated in a small number of fragments, forming a type 2 spot.

MS\#TPS0860.R1, - - LBNL-56776 -- 
A description of cathode spots composed of fragments and cells points to very small scales ( $\mu \mathrm{m}$ or less) and fast stages (some may be ns or faster). All measurements not resolving elementary events are composed of a multitude of stochastic processes. Stochastic processes involving dissipative structures are known to lead to fractals, which are self-similar on all scales down to the physical cutoff scale. Fractals are often related to power laws, which are always self-similar, i.e. invariant to multiplicative scaling.

The visual appearance of spot motion, i.e., the temporal and spatial distribution of ignitions, lead to previous suggestions of self-similarity. This was manifested in both patterns of emitted light and arc erosion craters. Further evidence for fractal properties was provided here by considering the noise behavior of ion current and arc voltage. Experimental power spectra showed characteristic brown or near-brown noise. Additionally, macroparticle distributions were also shown to be self-similar down to the resolution limit.

The fractal character of spots suggests to re-examine previous measurements and their interpretation. In particular, the current density appears to be better interpreted by a fractal than by the long-anticipated "true" value. Even with today's modern equipment, it is not yet clear where the physical small-scale cutoffs for the fractal current density, cell structure, and elementary process times lie.

\section{Acknowledgements}

The author would like to thank Efim Oks, Gera Yushkov, Konstantin Savkin, Peter Siemroth, Burkhard Jüttner and the late Ingmar Kleberg, who provided data and other input used for this paper.

MS\#TPS0860.R1, -- LBNL-56776 -- 


\section{REFERENCES}

[1] B. Jüttner, "Cathode spots of electrical arcs (Topical Review)," J. Phys. D: Appl. Phys., vol. 34, pp. R103-R123, 2001.

[2] R. L. Boxman, D. M. Sanders, and P. J. Martin, Ed., Handbook of Vacuum Arc Science and Technology. Park Ridge, N.J.: Noyes Publications, 1995.

[3] G. A. Mesyats, Cathode Phenomena in a Vacuum Discharge: The Breakdown, the Spark, and the Arc. Moscow, Russia: Nauka, 2000.

[4] B. B. Mandelbrot, The Fractal Geometry of Nature. New York: W.H. Freeman and Company, 1983.

[5] M. Schroeder, Fractals, Chaos, Power Laws: Minutes from an Infinite Paradise, 8th Ed., New York: W.H.Freeman and Company, 2000.

[6] E. Hantzsche, B. Jüttner, and H. Pursch, "On the random walk of arc cathode spots in vacuum," J.Phys.D: Appl.Phys., vol. 16, pp. L173-L179, 1983.

[7] J. E. Daalder, "Random walk of cathode arc spots in vacuum," J.Phys.D: Appl.Phys., vol. 16, pp. 17$27,1983$.

[8] R. P. P. Smeets and F. J. H. Schulpen, "Fluctuations of charged particle and light emission in vacuum arcs," J. Phys. D: Appl. Phys., vol. 21, pp. 301-310, 1988.

[9] S. Anders and B. Jüttner, "Influence of residual gases on cathode spot behavior," IEEE Trans. Plasma Sci., vol. 19, pp. 705-712, 1991.

[10] T. Schülke and P. Siemroth, "Vacuum arcs cathode spots as a self-similarity phenomenon," IEEE Trans. Plasma Sci., vol. 24, pp. 63-64, 1996.

[11] P. Siemroth, T. Schülke, and T. Witke, "Investigations of cathode spots and plasma formation of vacuum arcs by high speed microscopy and spectrography," IEEE Trans. Plasma Sci., vol. 25, pp. $571-579,1997$.

[12] I. G. Kesaev, Cathode Processes in the Mercury Arc (authorized translation from the Russian). New York: Consultants Bureau, 1964.

[13] J. M. Lafferty, Vacuum Arcs - Theory and Applications. New York: Wiley, 1980.

[14] G. A. Mesyats and D. I. Proskurovsky, Pulsed Electrical Discharge in Vacuum. Berlin: SpringerVerlag, 1989.

MS\#TPS0860.R1, - - LBNL-56776 -- 
[15] A. Anders, Cathodic Arc Plasma Deposition: From Fractal Spots to Energetic Condensation (in preparation). New York: Springer, 2006.

[16] M. J. Dryvesteyn, "Electron emission of the cathode of an arc," Nature, vol. 137, pp. 580, 1936.

[17] C. G. Suits and J. P. Hocker, "Role of oxidation in arc cathodes," Phys. Rev., vol. 53, pp. 670, 1938.

[18] J. D. Cobine, "Effects of oxides and impurities on metallic arc reignition," Phys. Rev., vol. 53, pp. $911,1938$.

[19] G. A. Lyubimov and V. I. Rakhovsky, "The cathode spot of a vacuum arc," Sov. Phys. Usp., vol. 21, pp. $693-718,1978$.

[20] J. Achtert, B. Altrichter, B. Jüttner, P. Pech, H. Pursch, H.-D. Reiner, W. Rohrbeck, P. Siemroth, and H. Wolff, "Influence of surface contaminations on cathode processes of vacuum discharges," Beitr. Plasmaphys., vol. 17, pp. 419-431, 1977.

[21] K. Jakubka and B. Jüttner, "The influence of surface conditions on the initiation, propagation and current density of unipolar arcs in fusion devices," J. Nucl. Mat., vol. 102, pp. 259-266, 1980.

[22] J. Halbritter, "On the contamination on electrode surfaces and electric field limitations," IEEE Trans. Elect. Insul., vol. 20, pp. 671-681, 1985.

[23] W. D. Davis and H. C. Miller, "Analysis of the electrode products emitted by de arcs in a vacuum ambient," J. Appl. Phys., vol. 40, pp. 2212-2221, 1969.

[24] I. G. Brown, "Vacuum arc ion sources," Rev. Sci. Instrum., vol. 65, pp. 3061-3081, 1994.

[25] M. P. Reece, "Burning voltage of arcs in high vacuum," Nature, vol. 181, pp. 475-476, 1958.

[26] A. Anders, B. Yotsombat, and R. Binder, "Correlation between cathode properties, burning voltage, and plasma parameters of vacuum arcs," J. Appl. Phys., vol. 89, pp. 7764-7771, 2001.

[27] A. Anders and G. Y. Yushkov, "Ion flux from vacuum arc cathode spots in the absence and presence of magnetic fields," J. Appl. Phys., vol. 91, pp. 4824-4832, 2002.

[28] E. Byon and A. Anders, "Ion energy distribution functions of vacuum arc plasmas," J. Appl. Phys., vol. 93, pp. 1899-1906, 2003.

[29] V. M. Lunev, V. G. Padalka, and V. M. Khoroshikh, "Use of a monopole mass spectrometer for investigating the ion component of a plasma stream generated by a vacuum arc," Instrum. Exp. Tech., vol. 19, pp. 1465-1467, 1976.

MS\#TPS0860.R1, - - LBNL-56776 - - 
[30] I. G. Brown, P. Spädtke, D. M. Rück, and B. H. Wolf, "Beam intensity fluctuation characteristics of the metal vapor vacuum arc ion source," Nucl. Instrum. Meth. Phys. Res. A, vol. 295, pp. 12-20, 1990.

[31] A. Anders, "Plasma fluctuations, local partial Saha equilibrium, and the broadening of vacuum-arc ion charge state distributions," IEEE Trans. Plasma Sci., vol. 27, pp. 1060-1067, 1999.

[32] G. A. Mesyats, "Ecton mechanism of the vacuum arc cathode spot," IEEE Trans. Plasma Sci., vol. 23, pp. 879-883, 1995.

[33] S. A. Barengolts, G. A. Mesyats, and D. L. Shmelev, "Structure and time behavior of vacuum arc cathode spots," IEEE Trans. Plasma Sci., vol. 31, pp. 809-816, 2003.

[34] I. I. Beilis, "Theoretical modeling of cathode spot phenomena," in Handbook of Vacuum Arc Science and Technology, R.L.Boxman, P. J. Martin, and D. M. Sanders, Eds., Park Ridge, N.J.: Noyes, 1995, pp. 208-256.

[35] H.-O. Peitgen and P. H. Richter, The Beauty of Fractals. Berlin: Springer, 1986.

[36] A. R. Osborne and A. Pastorello, "Simultaneous occurrence of low-dimensional chaos and colored random noise in nonlinear physical systems," Phys. Lett. A, vol. 181, pp. 159-171, 1993.

[37] P. Bak, C. Tang, and K. Wiesenfeld, "Self-organized criticality: An explanation of 1/f noise," Phys. Rev. Lett., vol. 59, pp. 381-384, 1987.

[38] S. Chandrasekhar, "Stochastic problems in physics and astronomy," Rev. Mod. Phys., vol. 15, pp. 189, 1943.

[39] G. F. Lawler, Introduction to Stochastic Processes. New York: Chapman \& Hall/CRC, 1995.

[40] B. Jüttner, V. F. Puchkarev, E. Hantzsche, and I. Beilis, "Cathode Spots," in Handbook of Vacuum Arc Science and Technology, R. L. Boxman, D. M. Sanders, and P. J. Martin, Eds. Park Ridge, New Jersey: Noyes, 1995, pp. 73-281.

[41] B. Jüttner, "The dynamics of arc cathode spots in vacuum: new measurements," J. Phys. D: Appl. Phys., vol. 30, pp. 211-229, 1997.

[42] B. Jüttner, H. Pursch, and V. A. Shilov, "The influence of surface roughness and surface temperature on arc spot movement in vacuum," J. Phys. D: Appl. Phys., vol. 17, pp. L31-L34, 1984.

MS\#TPS0860.R1, -- LBNL-56776 -- 
[43] J. E. Daalder, "Random walk of cathode spots: a random walk of definitions?," J.Phys.D: Appl.Phys., vol. 16, pp. L177-L179, 1983.

[44] V. V. Kraft and V. M. Stuchenkov, "Effect of nonmetallic inclusions in cathodes on vacuum breakdown," Sov. Phys -Tech. Phys., vol. 17, pp. 66-70, 1972.

[45] G. F. Lawler, Intersections of Random Walks. Boston, MA: Birkhäuser, 1991.

[46] K. Takeda and M. Sugimoto, "Effects of active elements on oxide removal by a vacuum arc," IEEE Trans. Plasma Sci., vol. 31, pp. 983-986, 2003.

[47] B. Jüttner, personal communication, 2004.

[48] T. A. Witten Jr. and L. M. Sander, "Diffusion-limited aggregation, a kinetic critical phenomenon," Phys. Rev. Lett., vol. 47, pp. 1400-1403, 1981.

[49] A. Anders, E. M. Oks, G. Y. Yushkov, K. P. Savkin, I. G. Brown, and A. G. Nikolaev, "Measurements of the total ion flux from vacuum arc cathode spots," IEEE Trans. Plasma Sci., vol. 33, pp. in preparation for no.5 (Special Issue, Sept. 2005), 2005.

[50] S. Anders, A. Anders, and B. Jüttner, "Brightness distribution and current density of vacuum arc cathode spots," J. Phys. D: Appl. Phys., vol. 25, pp. 1591-1599, 1992.

[51] A. Anders, E. M. Oks, and G. Yu.Yushkov, "Cathodic arcs: Fractal voltage and cohesive energy rule," Appl. Phys. Lett., vol. 86, pp. 211503-1-3, 2005.

[52] J. E. Daalder, "Components of cathode erosion in vacuum arcs," J. Phys. D: Appl. Phys., vol. 9, pp. 2379-2395, 1976.

[53] S. Anders, A. Anders, K. M. Yu, X. Y. Yao, and I. G. Brown, "On the macroparticle flux from vacuum arc cathode spots," IEEE Trans. Plasma Sci., vol. 21, pp. 440-446, 1993.

[54] P. Siemroth, K. Müller, M. Lukovnikova, and A. Anders, "Size distribution and acceleration mechanisms of macroparticles in vacuum arc plasmas," in preparation, 2005.

[55] A. Anders, S. Anders, B. Jüttner, W. Bötticher, H. Lück, and G. Schröder, "Pulsed dye laser diagnostics of vacuum arc cathode spots," IEEE Trans. Plasma Sci., vol. 20, pp. 466-472, 1992.

[56] B. Jüttner, "The dynamics of arc cathode spots in vacuum," J. Phys. D: Appl. Phys., vol. 28, pp. 516$522,1995$.

MS\#TPS0860.R1, -- LBNL-56776 -- 
[57] E. Hantzsche, "The arc cathode regime: An approach from basic laws," Contrib. Plasma Phys., vol. 27, pp. 293-308, 1987.

[58] R. V. Latham, High Voltage Vacuum Insulation. London and New York: Academic Press, 1981.

[59] A. F. Rogozin and R. P. Fontana, "Reactive gas-controlled arc process," IEEE Trans. Plasma Sci., vol. 25, pp. 680-684, 1997.

[60] R. P. P. Smeets, "The origin of current chopping in vacuum arcs," IEEE Trans. Plasma Sci., vol. 17, pp. 303-310, 1989.

[61] A. Anders, "Chopping effect observed at cathodic arc initiation," IEEE Trans. of Plasma Sci., vol. 28, pp. 1303-1304, 2000.

[62] V. F. Puchkarev and A. M. Murzakayev, "Current density and the cathode spot lifetime in a vacuum arc at threshold currents," J. Phys. D: Appl. Phys., vol. 23, pp. 26-35, 1990.

[63] D. L. Shmelev and E. A. Litvinov, "The computer simulation of the vacuum arc emission center," IEEE Trans. Plasma Sci., vol. 25, pp. 533-537, 1997.

[64] B. Jüttner and I. Kleberg, "The retrograde motion of arc cathode spots in vacuum," J Phys. D: Appl. Phys., vol. 33, pp. 2025-2036, 2000.

MS\#TPS0860.R1, -- LBNL-56776 -- 


\section{Figures}

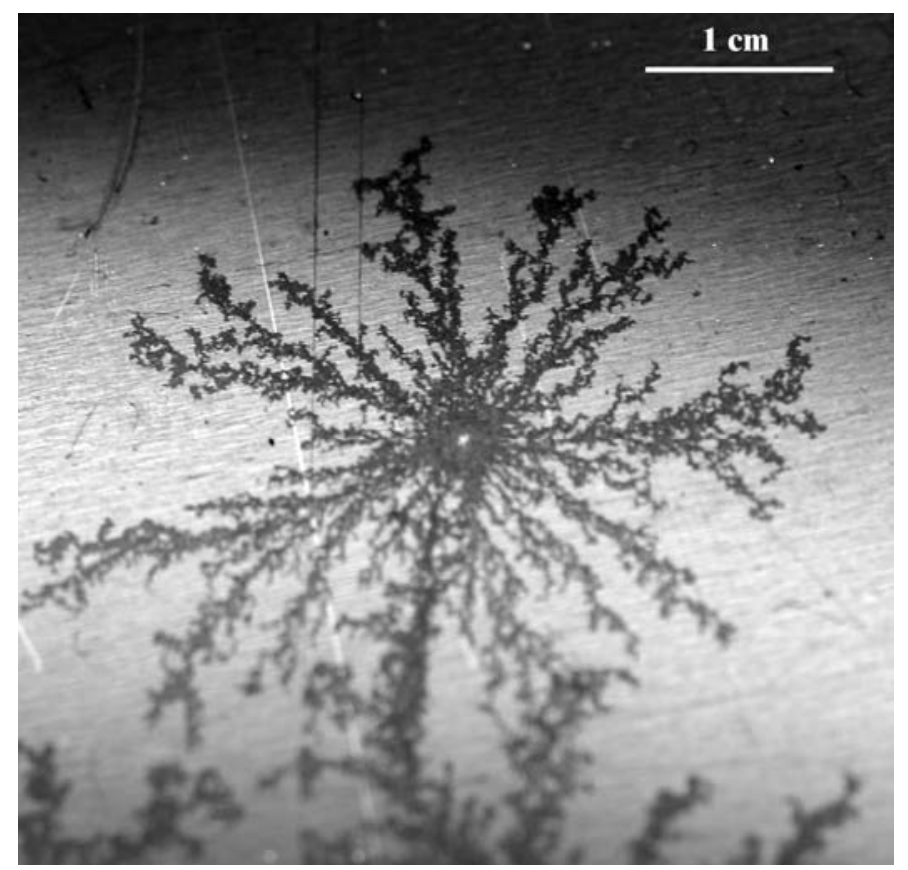

Fig. 1 Multi-spot arc traces on oxidized stainless steel. 


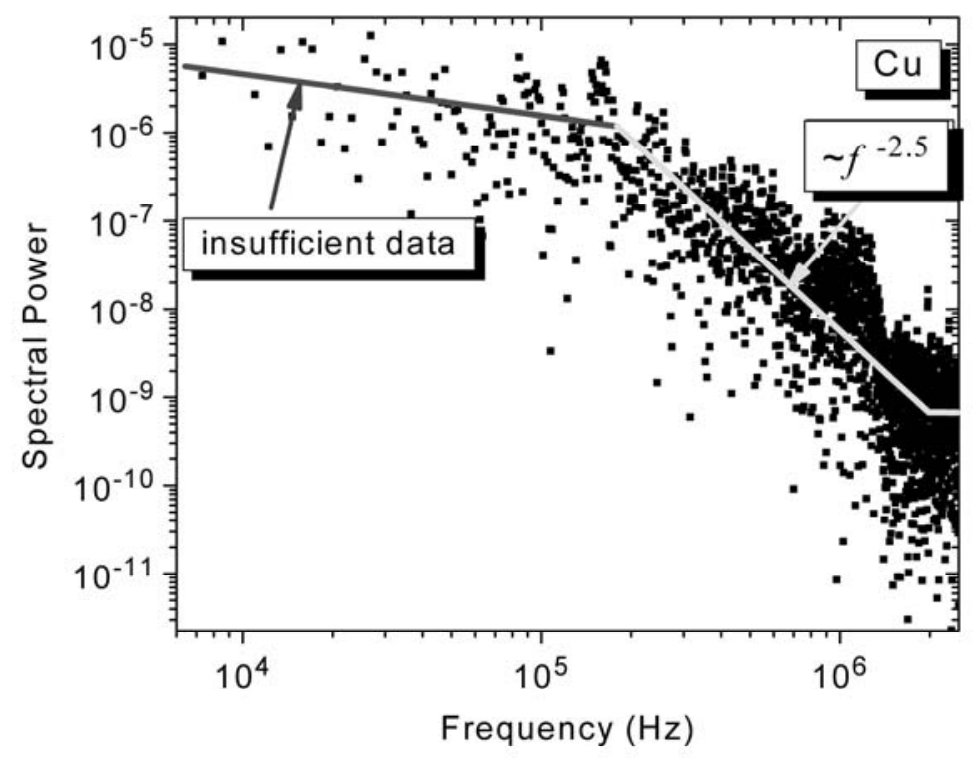

Fig. 2 Fast Fourier transform (power spectrum) of ion current for a copper vacuum arc (single pulse). One can see the approximate power law in the physically relevant interval. 


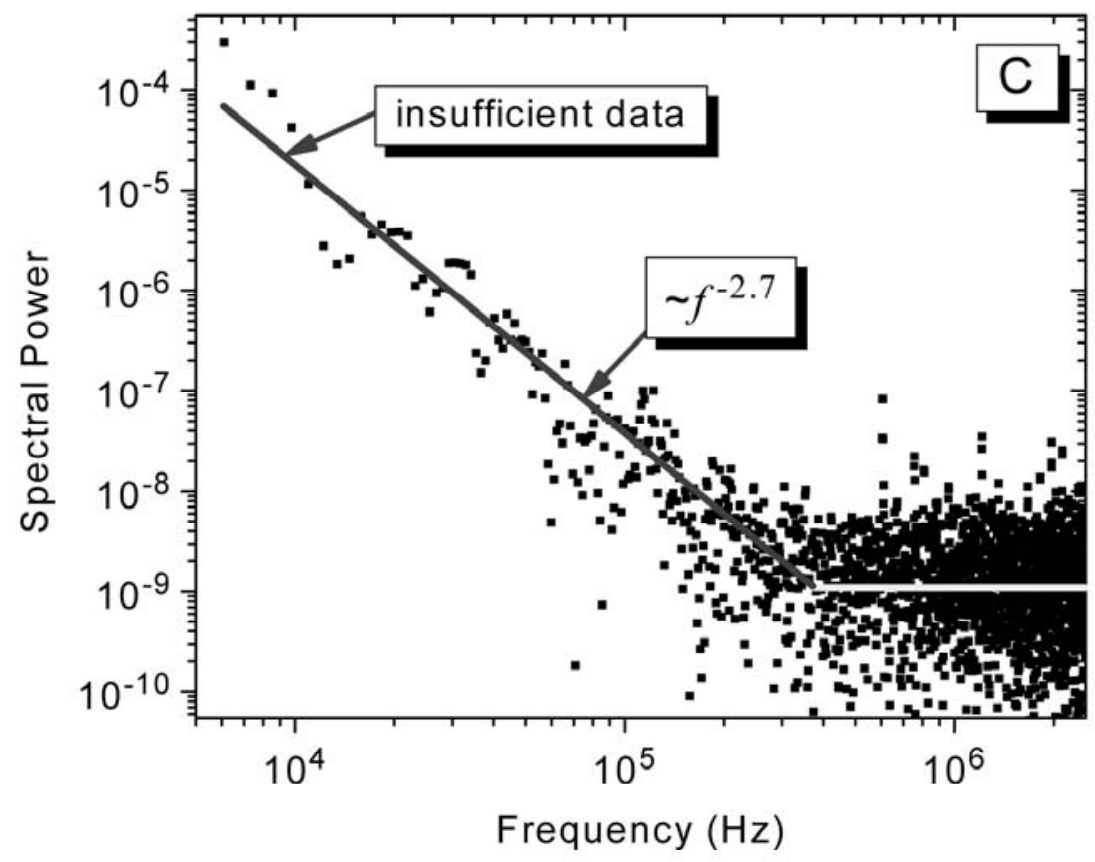

Fig. 3 As figure 2 but for a carbon arc pulse; the white noise extend to lower frequencies in this case. 


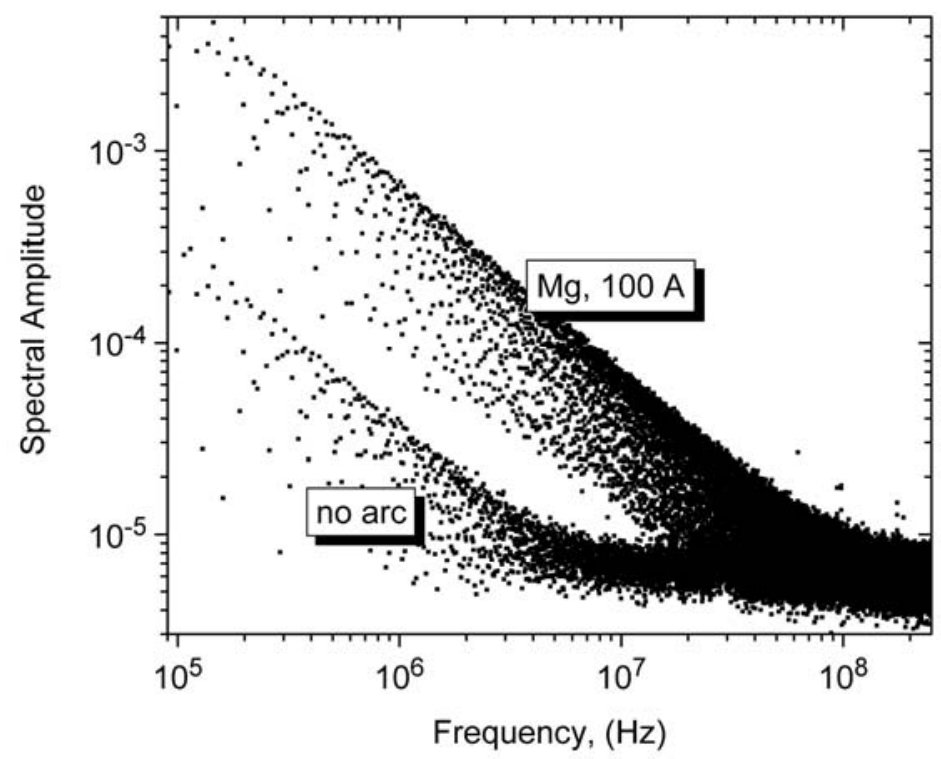

Fig. 4 Average of 16 FFT curves of the burning voltage measured for pulsed magnesium arcs $(100 \mathrm{~A}, 50,000$ points with 500 ps per data point in time domain). One can clearly see the power-law decay which is close to $1 / f$ in this amplitude presentation, corresponding to $\sim 1 / f^{2}$ for the spectral power. To indicate the reason for white noise at the high frequency end of measurements, the same FFT and average procedure was applied to the zero line (no arc). 


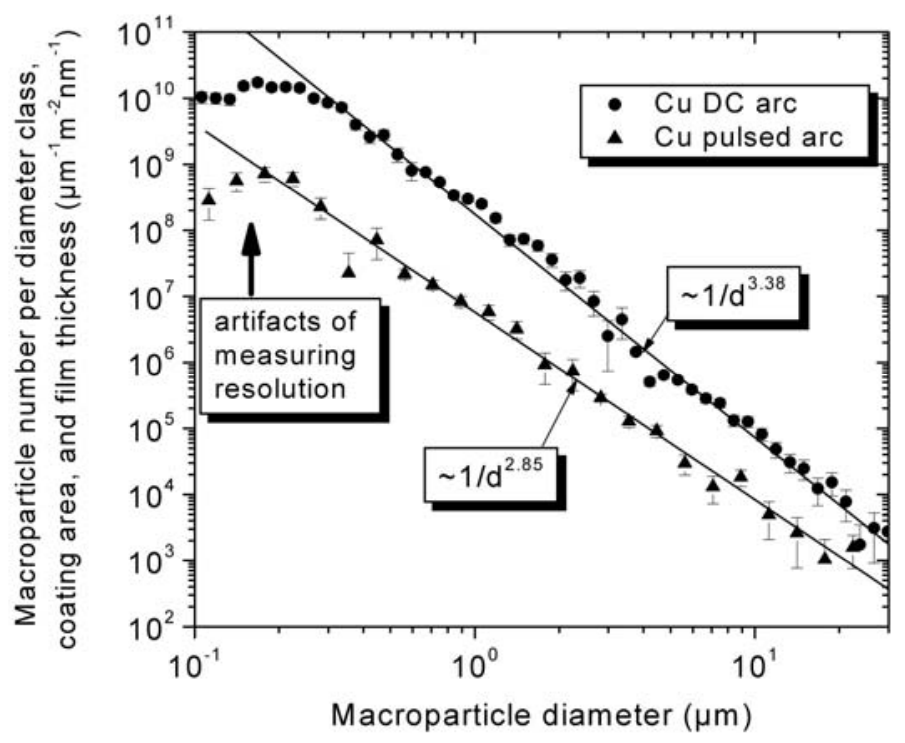

Fig. 5 Macroparticle size distribution for DC and pulsed copper arcs. The distributions show power laws indicating that a fractal dimension may be defined characterizing the dependence of the size distribution from experimental conditions. 


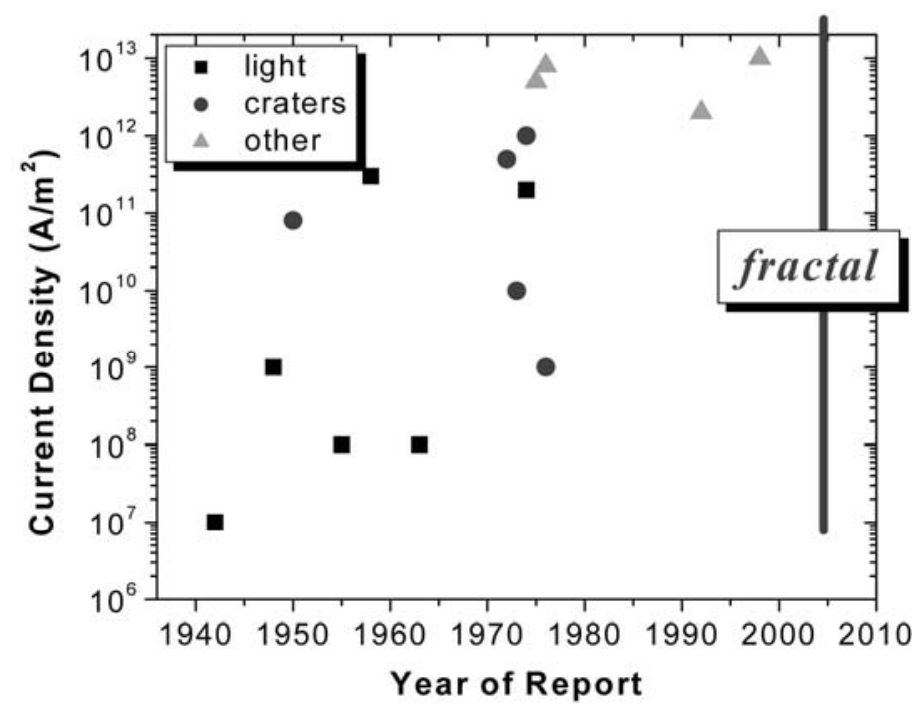

Fig. 6 Current density as measured over the years with various measuring techniques. Such plot was shown in the 1980s with a wink, in the assumption that one finally would settle on a value as techniques improved. Here it is argued that the current density of cathode spots is a fractal. 


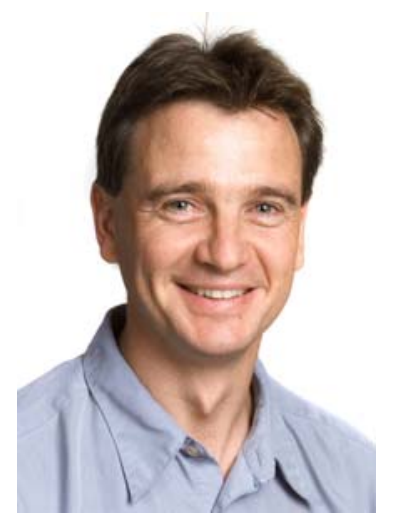

André Anders (M'94-SM'98-F'01) is a Senior Staff Scientist and the Leader of the Plasma Applications Group at Lawrence Berkeley National Laboratory, Berkeley, California. He studied physics in Wrocław, Poland, Berlin, Germany, and Moscow, Russia. He received a Ph.D. degree in physics in 1987 from Humboldt University, Berlin, Germany. From 1987 to 1991 he worked on electrode phenomena of arcs in gases and vacuum at the Academy of Sciences in Berlin. In 1992 he moved to Berkeley, California, where his research focuses on surface engineering and thin film deposition using plasma and ion beam methods. He is the author of A Formulary for Plasma Physics (Akademie, Berlin, 1990) and more than 150 scientific papers in refereed journals. He is the editor and co-author of the Handbook of Plasma Immersion Ion Implantation and Deposition (Wiley, NY, 2000). Currently he works on a book on cathodic arc deposition to be published by Springer, NY. He served as Guest Editor of IEEE Transaction on Plasma Science (1997, 2001). André is a Fellow of the Institute of Physics (UK), and member of the Editorial Board of Surface and Coatings Technology and of several international conference committees and research societies. He received the Chatterton Award in 1994 and an R\&D 100 Award in 1997.

MS\#TPS0860.R1, - - LBNL-56776 -- 